As Calvert says, vector manipulations are also not a fit precursor to decision or action. It is no more in order for a mariner in the midst of encounter to work them out than for a mathematician to reconstruct all the proofs of Euclid each time he wishes to use one of the theorems. Fortunately both exercises are equally as unnecessary as they are inappropriate. First, neither the situations of ships nor their actions are so various as to require it. Second, such manipulations as are required are readily done automatically, by a simple adjunct to the display device; or if desired, by a manual device not more elaborate or difficult to learn than the simplest sextant, less difficult to interpret than a cocked hat.

In the discovery role, there is but one field in need of further mathematical ploughing. It is the striking of optimum balances; between uncertainty and impotence, and between economy and safety, as mentioned above. In contrast to others, this field is not easy to plough, especially the second part. Nevertheless I think the yield will be well worth the effort, not only in safety itself but also in expedition without sacrifice of safety.

(I I) Perhaps I should add a second field for ploughing-the economic balance for collision avoidance. How much safety can maritime enterprise afford to buy? How much can it afford not to buy? I do not know. But I have heard it responsibly estimated that the money cost of Stockholm-Andrea Doria alone, direct and indirect, exceeded fifty millions of American dollars, net and irretrievable. Even today a tenth of this sum would buy a very great deal of excellent technology, plus quite a bit of its working product.

\title{
Mathematicians and Navigators
}

\author{
from E. S. Calvert
}

As Mr. Sadler points out, there can be no conflict between mathematicians and navigators, provided the mathematicians have adequately fulfilled their job. If, however, the navigators fail to follow the mathematics, or even to check the results by taking a few examples, then there will be plenty of misunderstandings; to bring 'philosophy' into the discussion is to return to the attitudes of the Middle Ages, and to obscure technical issues which are perfectly clear and simple.

Two of the contributors, Commander Clissold and Rear Admiral Gauw, do, however, make practical suggestions which are useful and interesting, and which require comment. Commander Clissold suggests that the proposed system of collision avoidance should be tested on a simulator. This system, like the existing one, applies, strictly speaking, to an encounter between two craft, and its correctness was demonstrated on a two-place radar simulator at the Royal Radar Establishment in 1959. The results were communicated to a meeting of the Technical Committee in January, 1961, and no one then or since has seriously questioned these results. It would therefore seem that what Commander Clissold has in mind, is an experiment which compares the two systems in various multiple situations, both qualitatively and quantitatively. After all, what 
matters in a given traffic pattern is the yearly collision rate at a given traffic density.

The writer therefore suggests that two groups of subjects, carefully matched as regards general ability, should be selected. One would be given the usual course of instruction in the existing regulations; the other would be given sets of mancuvring diagrams of the type proposed. Both groups would then be presented with various multiple situations, and their actions evaluated. This experiment would require a multi-place simulator, and would have to be conducted by people skilled in making such experiments, but costly as it might be, it is clear from the contributions in the Forum, that no progress will be made on the marine side until it is done.

Such an experiment would show that there are two distinct types of 'imponderables', if one must use such emotionally charged words in what should be a purely technical discussion. One type is due to the fact that, as Commander Clissold remarks, the existing Regulations give 'little specific guidance during the time between observing an echo and observing the ship which returns it'. Since the proposed system provides this guidance in a simple and absolutely unambiguous manner, and since the same manœuvres apply to both visual and radar sightings, the writer believes that this experiment would show that the particular "imponderables' associated with the defects of the present Regulations are not present in the proposed system. The other type of 'imponderable' is due to the inaccuracy of the means of detecting constancy of bearing, and no rules will remove these.

Another thing which the writer believes would be revealed is that the proposed system will work well so long as what one may call 'the mean free path' is large. As the density increases, one would expect that the first effect would be reductions in the manœuvring range and in the manœuvring displacement, and that eventually a stage would be reached when either the traffic slowed down, or the collision rate rose steeply. It is at this stage that it would become necessary to adopt a traffic structure such as Captain Oudet has proposed. The writer believes that the traffic density at which Captain Oudet's blue lines become desirable at sea will be higher for the proposed system, first because both vessels can manouvre, and secondly, because the manœuvres can be read off directly from a simple diagram without having to find closing speeds or aspects.

The writer would like to emphasize that the manœuvring range is taken into account in the manœuvring diagrams : since radar 'sees' through fog, the master of a radar-equipped vessel would not need to worry overmuch about the visibility were it not for the fact that he knows that there are at present no rules for the use of radar.

Rear Admiral Gauw submits five rules which he originally proposed in 1955, and seems to imply that his system is essentially the same as that proposed by the writer. No doubt the intention is the same, but the rules, as stated by Rear Admiral Gauw do not ensure that his intention will be implemented. When one of the two craft, say A, mancuvres, the line which must be made to rotate is not the actual line joining $A$ to the other craft, $B$. It is the line joining $A$ to the position which $B$ would occupy (at any moment) if $B$ had maintained course and speed. This line needs a name, as for instance, 'computed sight-line'. If the manœurres of $A$ and $B$ cause the two computed sight-lines to rotate in the same sense, then A will cross ahead of where $B$ would have been if it had not manœuvred, and B will cross astern of where A would have been if A had not manceuvred, or vice versa according to the convention adopted. In other words, the contributions 
are additive. Indeed, it is only by using this conception of two computed sight-lines that the contributions of each craft can be treated separately. If the mule of the road on land were stated in a form analogous to that which Rear Admiral Gauw proposes, it would require two vehicles meeting on a straight road (in the Netherlands) to pass left to left. A truck driver with no manners would be able to hog the crown of the road, no doubt maintaining that this was the ordinary practice of truck drivers. In other words, the rule could be satisfied even though the truck made no contribution at all to the miss distance; indeed, if the driver were thoroughly impolite, he might make a negative contribution, and force oncoming vehicles on to the verge. To prevent this, the rule of the road (in the Netherlands) is 'keep to the right,' and each vehicle infringes the mancuvring area of the other at its peril. The analogy is mathematically exact, as is made clear in Figs. $I$ and 2.

(X)

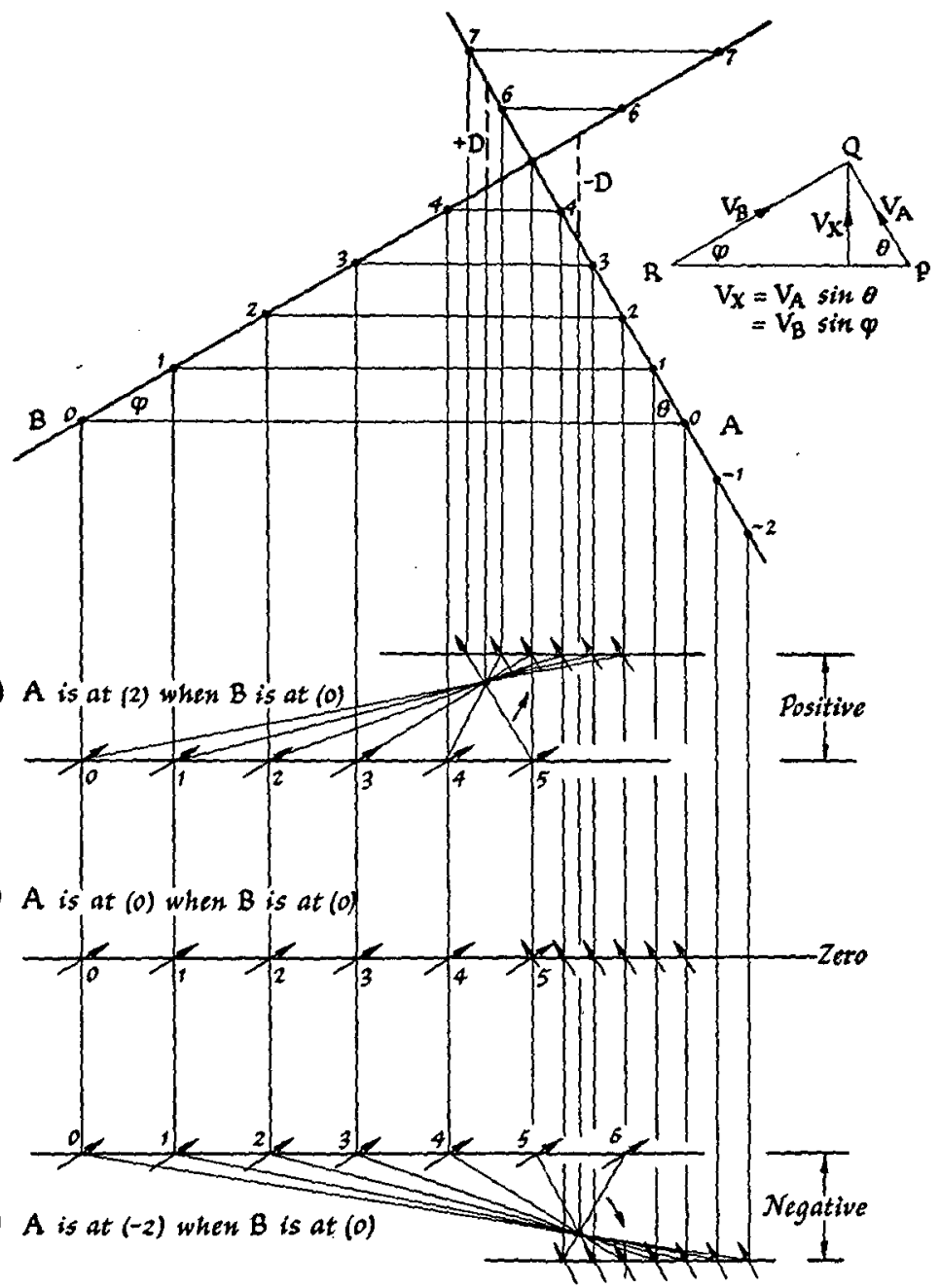

FIG. 1. Encounters with positive, zero and negative miss distances as seen in a frame of reference moving with velocity $v_{X}$ 
In these figures $A$ and $B$ are two craft with speeds and courses as shown in the triangle of velocities $P Q R$. A and $B$ will collide after the time $\left(t_{5}\right)$ if $A$ happens to be at $(A, 0)$ when $B$ is at $(B, 0)$. If $A$ happens to be at $(A, 2)$, then $B$, if it holds course and speed, will cross astern with a miss distance of $+D$. If $A$ happens to be at $(A,-2)$ when $B$ is at $(B, o)$, then $B$ will cross ahead, and the miss distance will be $-D$. If we now take a frame of reference moving with the velocity, $V_{X}$, equal to $V_{A} \sin \theta$ or $V_{B} \sin \varphi$, then these three encounters are as shown at $\mathrm{X}, \mathrm{Y}$ and $\mathrm{Z}$ in Fig. $\mathrm{I}$. In other words, by choosing a suitable frame of reference, all

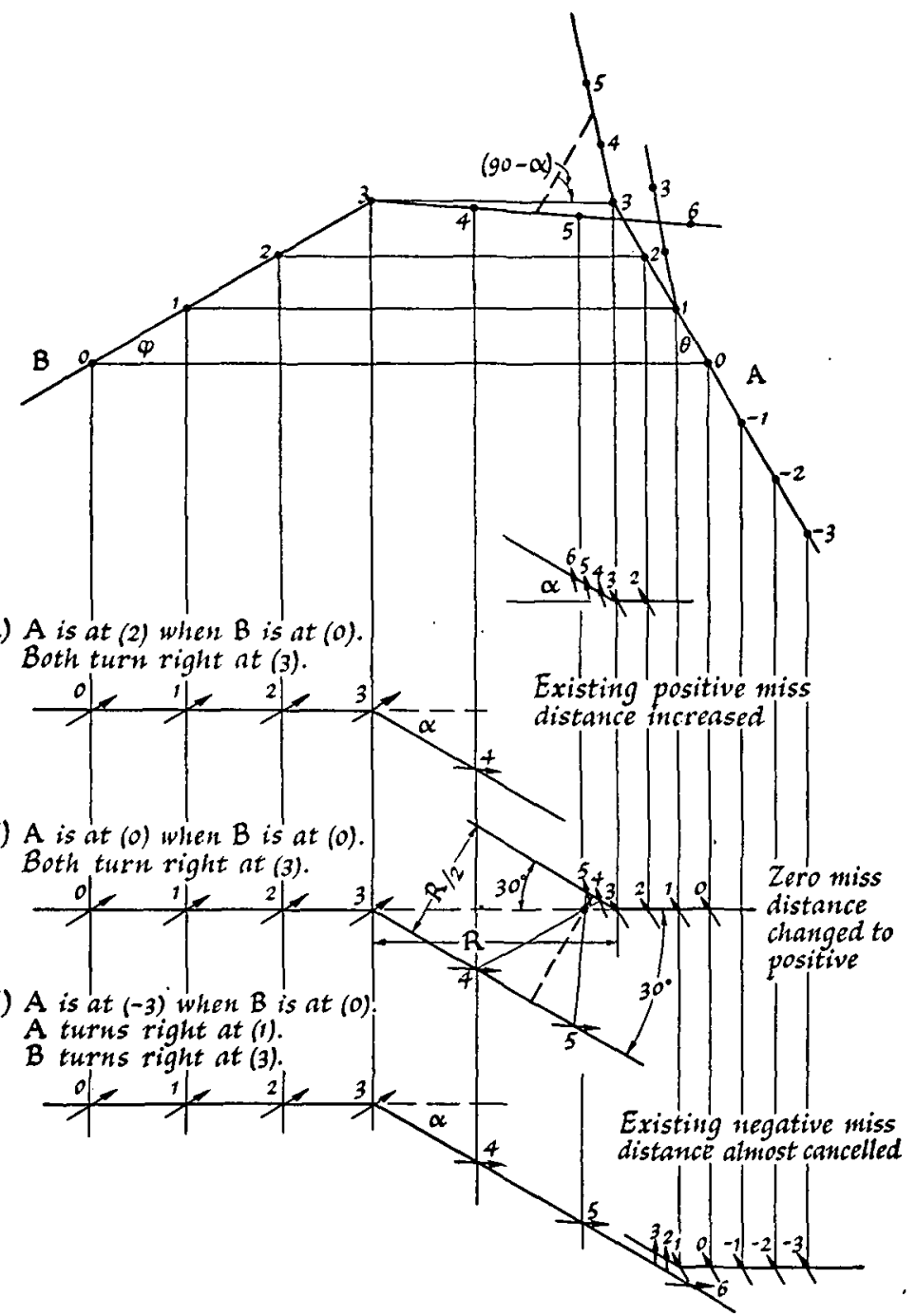

FIG. 2. Result of each craft applying normal manceuvres to the three types of encounter shown in Fig. $x$. The frame of reference moves with velocity, $V_{X}$, where $V_{X}=V_{A}$ $\sin \theta=V_{B} \sin \varphi$. In drawing these diagrams $\theta$ has been taken as $60^{\circ}$ and $\varphi$ as $30^{\circ} . A$ turns 18.6 and $B$ turns $34^{\circ} 3$, for which values $\alpha=30^{\circ}$. 
encounters can be regarded as taking place in a moving channel, and the safe manœuvring area appropriate to each craft is obvious. The difference between this concept and a real road is simply that encounters of the negative type should not occur, i.e. the markings in a real channel can be regarded as providing a detector of almost absolute accuracy. The head-on case is easily seen not to be a special type of encounter, but simply the particular case in which $V_{X}$ is zero, and the aspects are directly opposed.

Many of the difficulties of discussing this problem arise from the fact that the participants start with the collision encounter, which is rare, instead of with the close-quarter encounter, which is perhaps ten thousand times more frequent. They therefore consider only one set of manœuvres, the set which the writer has called 'positive'. Fig. 2 shows what happens when positive mancuvres are applied to the three types of encounter, $X, Y$ and $Z$. The alterations in course which cause the track of A or B to deviate by the angle, $\alpha$ (in the frame of reference), are given by formula $14(\mathrm{a})$ in Dr. Hollingdale's paper. When $\alpha$ is $30^{\circ}$, and both vessels alter course simultaneously, as shown at $(Y)$ in Fig. 2, then the miss distance is half the manœuvring range, $R$, and it is obvious that the speed ratio is not involved. This frame of reference can be used to demonstrate many other results of interest to mathematicians, and possibly of value to some mariners. Here the writer would merely point out that the type of encounter so vividly described at the end of Rear Admiral Gauw's contribution might in some cases be due, not to pig-headedness, but to the fact that one party, because of the inaccuracies in this method of detection, has assessed the encounter as either $(\mathrm{X})$ or $(\mathrm{Y})$, and the other as $(\mathrm{Z})$. No amount of 'blasting' and no kind of rules (assuming the traffic flow continues) will resolve this type of imponderable. With increasing accuracy in the means of detecting constancy of bearing, and a proper display, this type of situation will become rarer, but will never be completely eliminated in a random traffic structure. Incidentally, all these diagrams can be drawn without the use of the triangle of velocities merely by projecting positions from the 'true motion' diagram at the top of each figure. It is therefore hoped that mariners who wish to contribute to this discussion, but who cannot follow the mathematics, will do this for a number of encounters, and thereby satisfy themselves that the proposed manœuvres will always produce the effect which is claimed for them.

Rear Admiral Gauw says that his rules must never be reversed. Fig. 2, (Z), shows what this will lead to if the encounter is one in which the miss distance on the original courses is in fact negative. If Rear Admiral Gauw means that the convention should never be reversed in an encounter which each party assesses as a collision, then the writer strongly agrees, and this view is very clearly stated in Section ( 5 ) of his paper $\left(15,3^{88-90}\right)$. Actually, the writer has never used the term 'reversed rules'. The term 'reversed manocurres' refers to those (negative) manœuvres which produce a negative contribution, and which therefore increase a negative miss distance if this already exists. The existing rules permit both positive and negative manouvres, so 'reversal of the rules' in this case merely means the reversal of the preference for positive manœuvres. The fact that many mariners cannot see this reveals the confused thinking which results from the 'weasel wording' used in the existing Regulations. The essence of the writer's proposals is to stop the use of negative manœuvres in a collision situation, and thereby permit either or both vessels to manouvre. The whole point about this, and it is one which some of the contributors evade, is that it permits proper rules 
to be drawn up for the use of shipborne radar. This, of course, would take away the freedom of mariners to cancel each others' manceuvres, and this loss is, apparently, more than some of them can stomach. Airmen, on the other hand, take far more stringent controls as a matter of course, as Captain Oudet has so gently pointed out $(15,22)$.

With the exception of Commander Clissold, all the mariners who have written take it for granted that every proficient mariner knows what manœuvres will cause one vessel to cross ahead or astern of the other, particularly in an emergency. This really assumes that mariners in general have so absorbed the idea of relative motion that they can apply it quickly and correctly without conscious thought, but anyone who has watched subjects (mariners, airmen and mathematicians) guessing what to do on a simulator, knows that this is seldom the case. If the work done at R.A.E. produced no other result, it would have rendered a service to practising mariners in that it has enabled the manœuvres associated with a given end-result to be read off from a suitable diagram. This, according to some of the contributors, is help which many mariners do not want; some even resent it. Those who have been unfortunate enough to have been involved in a collision might, perhaps, have other views if they took the trouble to understand more fully the technical position.

Mathematicians, like mariners, are not perfect, and as Mr. Sadler remarks, may inadequately fulfil their job. In this context the common fault of the mathematicians has been that hitherto they have followed the mariners in trying to find a general solution by considering a series of particular encounters. $1 \AA$ recent example of this is the paper, 'The Physics of Collision at Sea', by Morrel $(\mathbf{1}, 165)$. In Fig. 5 of this paper, Ship (1) is shown as altering course $90^{\circ}$ to port; had the author been able to reduce the problem to the choice of one or other of the two possible conventions, he would have realized that this manœuvre is partly self-cancelling, i.e. the first $30^{\circ}$ causes the computed sight-line to rotate clockwise (negatively), the second $30^{\circ}$ cancels this rotation, and the last $30^{\circ}$ causes it to rotate anti-clockwise (positively). Actually, the same positive contribution could have been achieved more safely, and in a time about 2 minutes shorter, by a turn of $30^{\circ}$ to starboard, a fact which could be vital in an emergency. So long as the theory has not been fully worked out, it is possible that a shipload of mathematicians would do no better with radar sightings than mariners do now. However, as soon as the manœuvres have been reduced to a simple instruction diagram, even untrained personnel can use this to find out what contribution a given manœuvre will make far more quickly than the most expert navigator who has not this advantage. After all, the views of Aristotle about falling bodies were accepted uncritically for twenty centuries, but every schoolboy now knows that this illustrious philosopher was wrong.

\section{RE FER E N C E}

1 Miss your target reviewed in this Journal, 15,125 .

\section{Dr. S. H. Hollingdale comments:}

In his contribution to the current Forum, Mr. Calvert develops an analogy between collision avoidance at sea and on an ordinary road. To do this he employs a frame of reference which moves with the velocity $V_{X}$ of Fig. I; that is, the velocity component of either craft in a direction perpendicular to the 
relative velocity. Now Mr. Calvert states (see, for example, his Fig. 2) that if either craft makes a sharp change of course in accordance with formula (1 4a) of my paper $(\mathbf{1 4}, 253)$, then its track, in the chosen frame of reference, will turn through an angle $\alpha$. Note that nothing is said about the motion of the other craft.

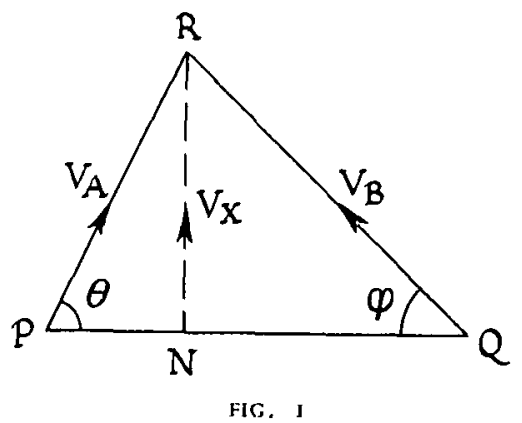

In my paper the angle $\alpha$ is defined in quite a different way; namely, as the angle through which the relative velocity vector rotates when both craft make sharp changes of course. It should be noted that the two craft need not be on a collision course; this definition of $\alpha$ applies equally well to a 'miss' situation, provided that no reference is made to the sight line. We are concerned only with velocity vectors, not with positions in space, nor with the times at which manœuvres are made.

The purpose of this note is to establish the truth of $\mathrm{Mr}$. Calvert's statement.

Fig. 1 shows the velocity triangle of craft $\mathrm{A}$ and $\mathrm{B}$ before manœuvre, with $\mathrm{NR}=V_{X}=V_{A} \sin \theta=V_{B} \sin \varphi$. (We assume that both $\theta$ and $\varphi$ are less than $90^{\circ}$.) The apparent velocities of the two craft in the chosen frame of reference are represented by PN and QN

Now let craft $A$ make a sharp turn through an angle $\alpha$ to starboard, its new velocity vector being represented by $P^{\prime} R^{\prime}$ in Fig. 2 . If we draw $R^{\prime} N^{\prime}$ equal and parallel to $R N$, then $P^{\prime} N^{\prime}$ will represent the new apparent velocity of craft $A$ in the chosen frame of reference. The manceuvre of craft $A$ thus causes its apparent track to turn through the angle marked $\beta$ in Fig. 2. The angle $R^{\prime} P^{\prime} N^{\prime}$ is $(\theta-\gamma+\beta)$. Now from triangle $P^{\prime} R^{\prime} N^{\prime}$, we have

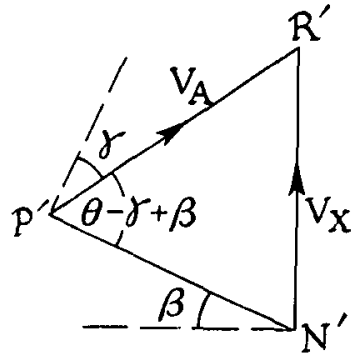

FIC. 2

$$
\frac{V_{A}}{\cos \beta}=\frac{V_{X}}{\sin (\theta-\gamma+\beta)}=\frac{V_{A} \sin \theta}{\sin (\theta-\gamma+\beta)}
$$

Hence

$$
\sin (\theta-\gamma+\beta)=\cos \beta \sin \theta
$$

Now if the change of course of craft $A$ is in accordance with formula (14a) of my paper, we have

$$
\sin (\theta-\gamma+a)=\cos \alpha \sin \theta
$$

A comparison of ( 1 ) and (2) shows at once that $\alpha=\beta$; that is to say, the apparent track of craft $A$, in the chosen frame of reference, turns through the angle $\alpha$. A similar argument can clearly be applied to craft B.

This proves Mr. Calvert's statement.

\section{Captain F.J. Wylie comments :}

I have read Dr. Morrel's comments with the greatest interest and pleasure. It is salutary to be dissected so gently. He takes me up a little wrongly on one or two points as, for example, when he implies that I dismiss all mathematical solutions and that I consider present day radar data to be adequate. 
Mr. Calvert's dismissal of philosophy and his very incomplete summing up of imponderables invite comment because they are fundamental to what seem to be our differences. However, no more is needed than a reference to the remarks of another mathematician, Mr. Sadler, on pages 105 and 106 of Volume 15, No. I of this Journal, where, with authority, diplomacy and obvious understanding, he describes the place of mathematics in this field; I agree entirely with his views.

I am glad to see that Mr. Calvert is emphasizing the need to deal, not only with steady-bearing cases, but also with the close approaches. The latter lend emphasis to the point made in my last note (15, 104) that, to be successful, a system based on manœuvring directive must contain clear indications of the circumstances in which the mariner ceases to have freedom of choice and must follow the directive.

I shall be very interested to hear Captain Oudet's reaction to a conjunction between the Calvert plan of manœuvre and his own traffic structure; at present $(15,19,20)$ he sees the life of the Collision Regulations continuing.

\title{
Ocean Routing Charts
}

\author{
from Captain A. F. Dickson \\ (Shell Tankers, Ltd.)
}

Passenger and cargo liners sailing on regular routes follow tracks for a given voyage, taking account of the various seasonal conditions which may apply, and masters of these ships, with many years experience in a particular trade, can decide without great difficulty the most advantageous track for their ship when planning a voyage for a given time of year.

In the transport of oil, on the other hand, it is not common to employ ships on regular voyages, and the great majority of tankers are employed in world-wide trading, so that although a particular oil company may have a number of ships constantly voyaging on a particular route, a high degree of flexibility is maintained and ships are regularly changed from one route to another. This means that the tanker master is called upon to consider the requirements for optimum routing of his ship on any ocean voyage. Further, he is often given his orders with fairly short notice and, in some cases, he may have his destination changed either at the last moment or during the voyage, leaving him little time to consider the many factors which have to be taken into account when planning the best route for the voyage to be undertaken.

Bearing this in mind, some thought has been given to the way in which choice of route can be facilitated. It is suggested that when planning an ocean voyage, consideration has to be given to the following:

(1) The navigational limitations which apply to the ship in question. For example, when planning a voyage for a deep-draft tanker from Borneo to South Australia, it would have to be found out whether the depths in the Torres Straits were sufficient to allow the ship to use this passage. 